\title{
Terroir of yeasts? - Application of FTIR spectroscopy and molecular methods for strain typing of yeasts
}

\author{
Daniel Gerhards ${ }^{1}$, Nicole Büchl ${ }^{2}$, Mareike Wenning ${ }^{2}$, Siegfried Scherer ${ }^{2}$, and Christian von Wallbrunn ${ }^{1}$ \\ ${ }^{1}$ Institute for Microbiology and Biochemistry, Hochschule Geisenheim University, Von-Lade-Str.1, 65366 Geisenheim, \\ Germany \\ ${ }^{2}$ Department of Microbiology, ZIEL - Zentralinstitut für Ernährungs- und Lebensmittelforschung, TU München, \\ Weihenstephaner Berg 1, 85350 Freising, Germany
}

\begin{abstract}
The site specific influence on wine (Terroir) is an often by wine producers, consumers and scientists discussed topic in the world of wine. A study on grapes and (spontaneous) fermentations from six different vineyards was done to investigate the biodiversity of yeasts and to answer the question if there is a terroir of yeast and how it could be influenced. Randomly isolated yeasts were identified by FTIR-spectroscopy and molecular methods on species and strain level. Vineyard specific yeast floras would be observed but they are not such important as expected. Only a few overlapping strain patterns would be identified during both vintages. The yeast flora of the winery had a huge impact on the spontaneous fermentations, but is not really constant and influenced by different factors from outside.
\end{abstract}

\section{Introduction}

The site specific influence on wine (Terroir) is an often discussed topic of wine producers, scientists and consumers. Originally this term "Terroir" was defined as specificity of different types of soil ("Le gout de terroir" - the taste of soil). According to the last definition by the OIV this term includes the whole of rock, soil, terrain, water balance, macro and micro climate as well as the work of the winemaker in vineyard and cellar. Many studies are known about the relationships between different sets and produced wines. But information about site-specific differences of microorganism, especially yeasts, occurring in the vineyard and their influence on (spontaneous) fermentations are rare. This was part of investigations of a FEI project (AiF $16008 \mathrm{~N}$ ): How site specific are yeast populations in vineyards and which influence have these populations on the quality of spontaneous fermented wines? Starting in the vineyards up to the processing of grapes and alcoholic fermentations yeast population were analysed.

\section{Material and methods}

This FEI project was focused on the processing of Riesling grapes and six vineyards representing three different soil types (loess, clay and slave) were included. Grape material and the resulting wines were analysed regarding to the biodiversity of yeasts and the sensorial and chemical composition. At different time points starting in the vineyards up to the end of fermentations samples of 100 yeasts were taken randomly. The identification of randomly isolated yeasts was done by FTIR (Fourier-transform infrared) spectroscopy. After standardized cultivation on YGC-Agar the middle infrared range of 25.000-2.500 nm was used to get specific absorption spectra of the whole living cells which are used for the species identification (see Fig. 1).

This method could be also an opportunity to discriminate yeasts on strain level. It is an interesting tool for typing on strain level because it considers the physiological state of a living yeast cell at a defined stage of growing under standardized conditions. The generated spectra are used to do hierarchical cluster analysis (HCA). Dendrograms are calculated by the Average Linkage Algorithm. The classification into sub-clusters is done by defining a spectral distance of 0.3 as a value for separation on strain level [1].

Also for the strain typing of selected isolates molecular techniques (RAPD and Delta-PCR) were used to compare the methods. For Hanseniaspora uvarum isolates RAPD analysis were done using the OPA-03 and OPA-18 primers.

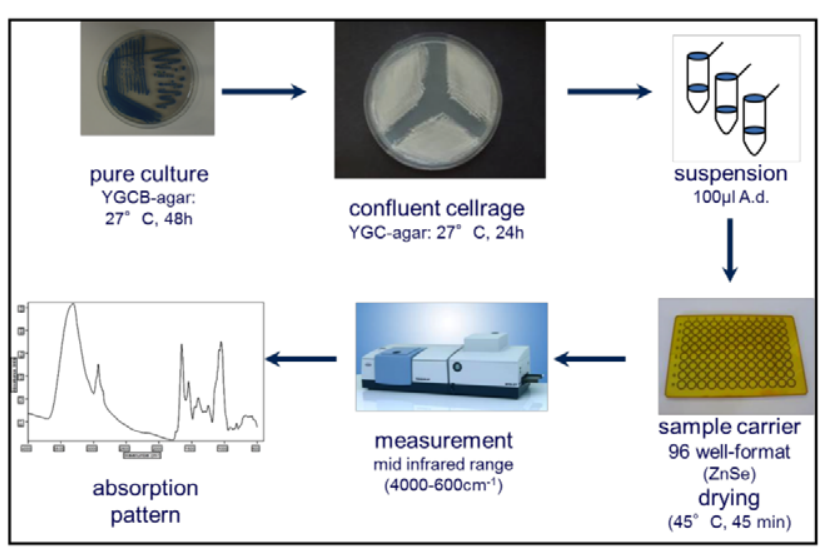

Figure 1. Standardised identification of yeast by FTIR spectroscopy. 
For differentiation of Saccharomyces cerevisiae strains a rapid PCR-based protocol, relying on the amplification of interdelta regions, was used according to Legras and Karst [2] to show polymorphisms on strain level. Additionally analytical (FTIR, HPLC, GC-MS) and sensorial data were determined.

\section{Results}

As a result a great biodiversity and distribution of yeast species and strains could be observed for all of the different samples (see Fig. 2).

During the duration time of two years of the spontaneous fermentation project only one strain of Hanseniaspora uvarum was present in both years if RAPD PCR using the OPA-3 and OPA-18 was done for the analysis on strain level (see Fig. 3).

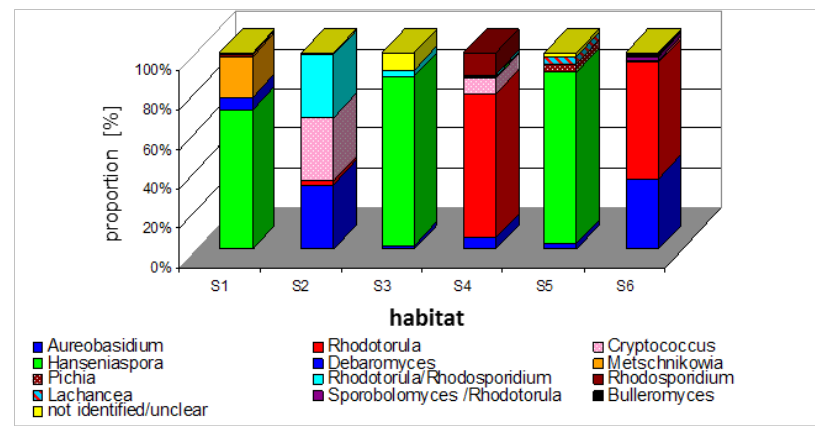

Figure 2. Representative biodiversity of yeast at the time point of harvesting for six different vineyards based on 100 randomly isolated yeasts for each sampling point.

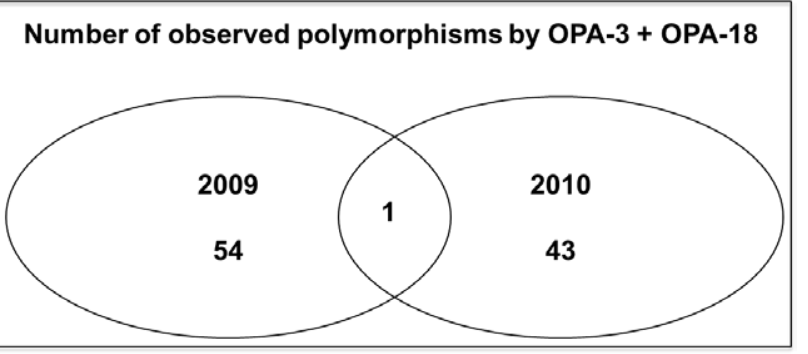

Figure 3. Year-on-year comparison of $H$. uvarum RAPD polymorphisms by OPA-03 and OPA-18.
Table 1. Differentiation patterns by $\delta$-PCR of $S$. cerevisiae strains isolated in 2019 and 2010.

\begin{tabular}{ll}
\hline 2009 & 2010 \\
\hline patterns & patterns \\
1 & 1 \\
Oenoferm Freddo & Oenoferm Freddo \\
3 & 3 \\
4 & 18 \\
5 & 19 \\
6 & 20 \\
7 & 7 \\
8 & 21 \\
9 & 22 \\
10 & 23 \\
11 & 24 \\
12 & 4 \\
13 & 25 \\
14 & 28 \\
16 & 29 \\
17 & 30 \\
& 31 \\
& 32 \\
& 33 \\
& 34 \\
& 35 \\
& 36 \\
$\sum 17$ (155 isolates) & 37 \\
\multicolumn{2}{c}{ Overlapping patterns: 5} \\
\hline
\end{tabular}

Only a few strain patterns of Saccharomyces cerevisae were present at both harvest times if the interdelta PCR was done for strain discrimination (see Table 1).

\section{Conclusion}

In conclusion FTIR spectroscopy is a suitable technique for discrimination of yeasts on strain level. The yeast flora of the vineyard could be identified, but was not such as sitespecific as expected. The occurring yeast flora of a winery has a big influence. This yeast flora of a winery would be influenced from outside and is not constant. Yeasts having a selective advantage will survive, but not necessarily the wished, strong fermenting and aroma specific ones.

Parts of this research project were supported by the German Ministry of Economics and Technology (via AiF) and the FEI (Forschungskreis der Ernährungsindustrie e.V., Bonn). Project AiF $17180 \mathrm{~N}$.

\section{References}

[1] C. Grangeteau, D. Gerhards, S. Rousseaux, C. von Wallbrunn, H. Alexandre, M. Guilloux-Benatier, Food Micro 50, 70-77 (2015)

[2] J.L. Legras, F. Karst, FEMS Microbiol Lett 221(2), 249-255 (2003) 